\section{La actividad de la OPS en el campo de las enfermedades no transmisibles ${ }^{1}$}

1 Extractado de "Noncommunicable Diseases" (CE120/18), documento de trabajo preparado por el Programa de Enfermedades No Transmisibles (HCN) para presentación por el doctor George A. O. Alleyne, Director de la Oficina Sanitaria Panamericana, a la 28a reunión del Subcomité de Planificación y Programación del Comité Ejecutivo de la Organización Panamericana de la Salud (abril de 1997) y revisado por el Comité Ejecutivo en su 120. a Reunión (junio de 1997).

2 Puffer RR, Griffith GW. Características de la mortalidad urbana: informe de la Investigación Interamericana de Mortalidad. Washington, DC: Organización Panamericana de la Salud; 1968. (Publicación científica 151).

3 Las enfermedades transmisibles, muertes maternas y causas perinatales completan el total con un porcentaje combinado de $32,3 \%$.
La Investigación Interamericana de Mortalidad llevada a cabo entre 1962 y 1964 reveló el impacto progresivo que estaban teniendo las enfermedades no transmisibles (ENT) en las poblaciones de las Américas. $^{2}$ Se publicaron al mismo tiempo estudios internacionales de importancia crítica, por ejemplo, la investigación de Framingham en los Estados Unidos de América y otras, que mostraban la posibilidad de modificar ciertos factores de riesgo y, con ello, de prevenir esas enfermedades. La cooperación técnica de la OPS en este campo, que en esa época se limitaba a ciertos aspectos de la epidemiología del cáncer, fue expandiéndose poco a poco y a mediados de los años setenta incluía la hipertensión, la diabetes y otras enfermedades. Para esa época se establecieron el primer Programa de Enfermedades Crónicas de la OPS, más tarde llamado Programa de Salud del Adulto, y unidades de enfermedades no transmisibles en los ministerios de salud de algunos Estados Miembros. Originalmente el interés se había centrado en la observación, pero el conocimiento de los resultados de nuevos estudios durante los años ochenta impulsó el planeamiento de programas de intervención. En los años noventa las prioridades del programa se han redefinido para incluir las enfermedades cardiovasculares, el cáncer y la diabetes, a las cuales se han incorporado las lesiones y discapacidades causadas por traumatismos.

El actual Programa de Enfermedades No Transmisibles (HCN) se inició en abril de 1995 como parte de la División de Prevención y Control de las Enfermedades y sustituyó el anterior Programa de Salud del Adulto. Su misión es potenciar la capacidad de la OPS para respaldar a los Estados Miembros en la prevención y el control de ciertas enfermedades crónicas y traumatismos. Las ENT desempeñan un papel cada vez más importante dentro del marco de la carga general de enfermedades en América Latina y el Caribe. Según las estadísticas de 1990, las ENT generan 57,9\% de aproximadamente 3 millones de muertes anuales por todas las causas y los traumatismos, $9,8 \%{ }^{3}$ Del total de muertes por ENT, las enfermedades cardiovasculares producen $45,4 \%$, los neoplasmas malignos $19,7 \%$ y la diabetes, $4,9 \%$.

Se prevé que la tendencia al aumento proporcional de las ENT seguirá manifestándose entrado el siglo XXI, debido a incrementos en la prevalencia 
de factores de riesgo, envejecimiento de las poblaciones, disminución de la fecundidad y control eficiente de las enfermedades contagiosas. Según las proyecciones, las defunciones por ENT, que en 1985 eran 1,5 veces más frecuentes que las defunciones por enfermedades infecciosas y parasitarias, habrán aumentado a una frecuencia 3,4 veces mayor en el año 2000 y 6,7 mayor en el 2015 (figura 1). Las ENT sobresalen también como causa principal de años de vida potencial perdidos por discapacidad y una estimación conservadora les atribuye $54,2 \%$ de ese total, mientras que a los traumatismos corresponden $17,7 \%$. Es evidente, por lo tanto, que urge cooperar con los sistemas de salud nacionales y facilitar su obtención de una capacidad de servicios suficiente para hacer frente a esas causas de defunción y discapacidad.

\section{Mitos y realidades}

Los conocimientos biomédicos más recientes revelan que muchas de las creencias tradicionales relacionadas con las enfermedades no transmisibles son mitos sin fundamento. Los análisis de la configuración de esas enfermedades en América Latina y el Caribe muestran que no son inevitables, ni exclusivas de la clase pudiente, ni predominantemente de la tercera edad. Más de $65 \%$ del total de los años de vida potencial perdidos por discapacidad debida a ENT corresponden a personas menores de 45 años y cerca de $30 \%$ a menores de 15 .

FIGURA 1. Razones entre defunciones por causas no transmisibles y por enfermedades infecciosas (DCNT:DEI) en América Latina y el Caribe

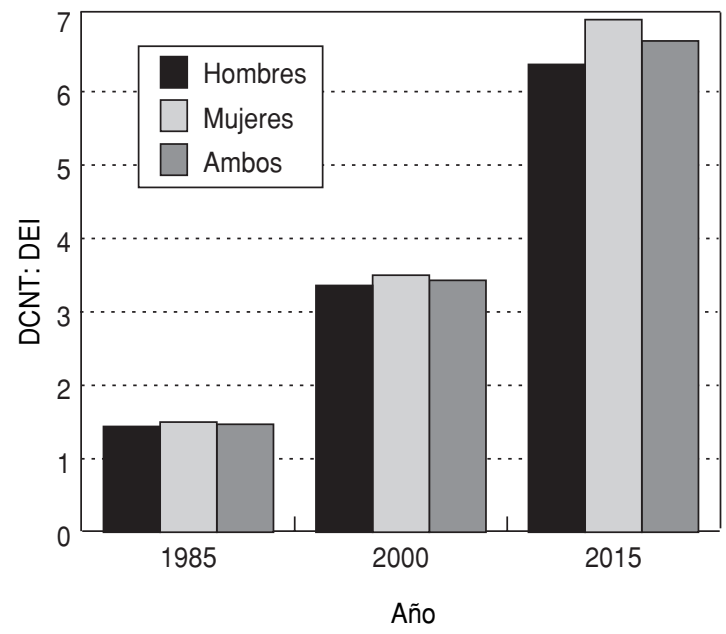

Fuente: Bulatao R, Stephens P. En: Gribble JN, Preston SH, eds. The epidemiological transition. Washington, DC: National Academy Press; 1993.
Ahora bien, si se consideran los años de vida potencial perdidos por discapacidad debida a todas las causas en menores de 45 años, las ENT dan cuenta de $44 \%$ y los traumatismos, de $21 \%$.

La asociación de las ENT con los estratos más pudientes de la sociedad parece haber sido una mala interpretación de su aumento coincidente con las tendencias hacia la industrialización y la transición demográfica. Incluso ha habido observadores acríticos que consideran el auge de las ENT indicador de progreso social. Por cierto, en la mayor parte de las ENT de importancia para la salud pública, la incidencia y la prevalencia de factores de riesgo son más altas en los bajos estratos socioeconómicos.

Por otra parte, tampoco es cierto que es imposible luchar contra ellas. En este sentido es bueno recordar que, antes de la vacuna antisarampionosa, muchas personas se referían al sarampión como una enfermedad de la niñez "inevitable y sin consecuencia". Asimismo es arcaica la frase "enfermedades crónicas degenerativas" y ya no debe usarse, pues implica irreversibilidad. En realidad, casi todas las dolencias así llamadas pueden prevenirse o posponerse, muchas son curables y otras susceptibles de prevención secundaria a fin de reducir las complicaciones y la pesada carga que imponen sobre los servicios de salud. La enfermedad isquémica del corazón, incluidas las lesiones ateroscleróticas, puede revertirse mediante el tratamiento apropiado de factores de riesgo como la hipertensión y la hiperlipidemia. En cuanto al cáncer, nunca ha sido degenerativo sino neoplásico, y la diabetes mellitus no insulinodependiente es relativamente reversible. Si bien es cierto que algunas afecciones como la osteoartritis son degenerativas y otras como las lesiones de la columna vertebral son irreversibles, el término más general de enfermedades crónicas abarca mejor al grupo.

\section{Enfermedades prioritarias}

Enfermedades del aparato circulatorio. En el Canadá y los Estados Unidos de América, a partir de 1980 se han conseguido reducciones proporcionales de alrededor de $15 \%$ de la mortalidad por estas enfermedades. Esas reducciones guardan relación con las disminuciones de prevalencia de los factores de riesgo principales, es decir con el hábito de fumar, la hipertensión y las hiperlipidemias. Esto no se ha logrado en América Latina y el Caribe, zonas donde los factores de riesgo van más bien en aumento.

Los determinantes que originan esos factores de riesgo, como las dietas ricas en grasas, el sedentarismo y el abuso de sustancias, tienen que ver con hábitos que se establecen temprano en la vida y que 
requieren modificación mediante estrategias de promoción de la salud y prevención primaria. Sin embargo, esas estrategias solo surten efecto a mediano o largo plazo. A corto o muy largo plazo es esencial prestar atención a la prevención secundaria, la prevención clínica y la calidad de la atención de los enfermos.

Cáncer. Desde el principio de los años sesenta hasta fines de los ochenta, las defunciones por cáncer en las Américas aumentaron 73\%. La mortalidad proporcional por cáncer se ha elevado en todos los países. En América Latina y el Caribe los cánceres más frecuentes son de cuello uterino, estómago, orofaringe, esófago, mama, pulmón, hígado y colorrecto, y linfomas y leucemias. El cáncer de estómago está disminuyendo, pero los de mama y pulmón $-\mathrm{y}$ de otros sitios afectados por el tabaquismo- siguen aumentando.

Cáncer de cuello de útero. El cáncer cervicouterino tiene tasas tan altas que su prevención es la intervención de prioridad para el Programa HCN. La mayor parte de los casos están asociados con el virus del papiloma humano, que se transmite ampliamente por vía sexual. El uso de condones ayuda en la prevención primaria, pero hasta ahora solo la prevención secundaria ha sido efectiva. Esta involucra la detección de alteraciones displásicas o cancerosas incipientes mediante pruebas citológicas, seguida de investigación, diagnóstico y tratamiento. Las personas encargadas de tomar decisiones necesitan saber que $60 \%$ del cáncer invasor ocurre en mujeres de 35 a 60 años de edad y que, por lo tanto, sobra justificación para acometer su prevención lo más enérgicamente posible.

El tamizaje del cáncer cervicouterino tiene la ventaja adicional de que permite abordar la salud general de la mujer, ya que este tipo de detección le da la oportunidad de tener acceso a los centros de salud, donde puede consultar sobre otras dolencias como hipertensión y diabetes. Hasta ahora, ese tamizaje se ha introducido casi sin excepción en los programas de salud materna y planificación familiar. Como consecuencia, los recursos disponibles se están aplicando casi exclusivamente y a intervalos demasiado cortos, por lo general anualmente, a mujeres saludables de bajo riesgo. Así se pierde la oportunidad de aplicar la prueba de detección a mujeres de edad mediana y de alto riesgo, que constituyen la mayoría avasalladora de casos de cáncer invasor. Para que esa detección sea más efectiva, se están promoviendo el tamizaje menos frecuente (por ejemplo, cada 5 años) en mujeres de 30
FIGURA 2. Uso de la morfina (mg/cápita) para aliviar el dolor del cáncer en ocho países de América Latina y el Caribe, 1993

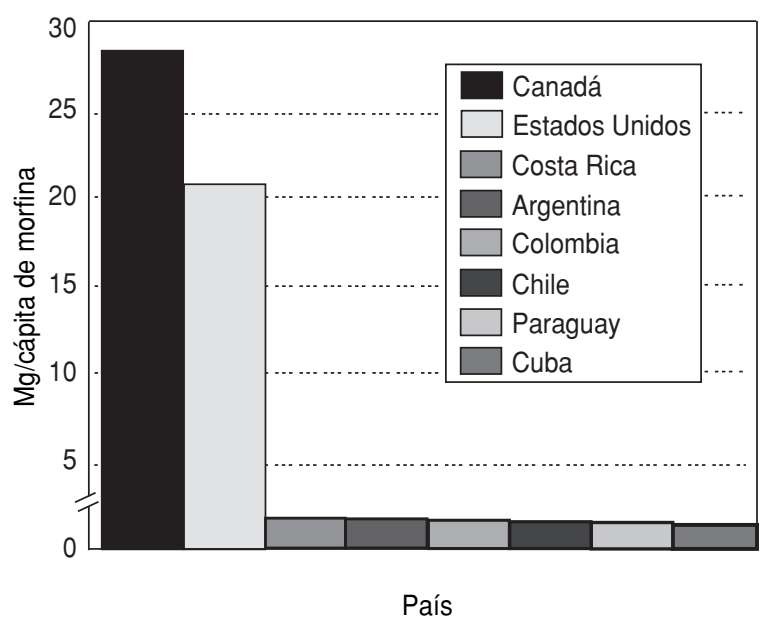

Fuente: OPS, Programa de Enfermedades No Transmisibles, Proyecto de Cuidado Paliativo.

a 60 años de edad y una cobertura más amplia que abarque a la mayoría del grupo en riesgo.

Alivio del dolor. Es de esperar que, al inicio de una promoción más intensa del tamizaje del cáncer en América Latina y el Caribe, se descubran muchos casos inoperables. Hay que anticipar que el manejo ético de esos casos requiere cuidados paliativos y alivio eficaz del dolor. En una encuesta reciente sobre el alivio del dolor en el cáncer, se usó un indicador basado en el uso de la morfina (figura 2). Mundialmente, el mejor índice correspondió al Reino Unido y, en la Región de las Américas, al Canadá. En cambio, los países encuestados en América Latina y el Caribe prácticamente no cuentan con medios para tratar el dolor intenso. Recientemente el Programa de la OMS sobre el Cáncer ha abordado este problema con éxito en el sudeste asiático y es importante que los países de las Américas se familiaricen con los progresos logrados en el tratamiento del dolor y el cuidado paliativo en el cáncer. La OMS ha publicado una monografía sobre el tema que está disponible en español. ${ }^{4}$ En estrecha consulta con el Programa Regional de Bioética y organizaciones como la Comisión Interamericana contra el Abuso de Drogas (CICAD) y mediante financiamiento del Consejo de Alberta (Canadá) sobre el Cáncer, el Pro-

\footnotetext{
4 Organización Mundial de la Salud. Alivio del dolor en el cáncer: con una guía sobre la disponibilidad de opioides. 2a ed. Ginebra: OMS: 1996
} 
grama HCN ha emitido un contrato para asistir en la elaboración de una propuesta de estrategia regional con respecto a cuidados paliativos y alivio del dolor intratable causado por el cáncer.

La cuestión de los registros de cáncer. El Programa $\mathrm{HCN}$ considera que los registros no son necesariamente ventajosos en todas las circunstancias. Si se instalan correctamente, pueden proveer información sobre tendencias y distribución de enfermedades muy útil para establecer prioridades y para diseñar, administrar y evaluar programas de cáncer. Lamentablemente, muchos registros no se instalan ni se mantienen adecuadamente porque con frecuencia se subestiman los recursos necesarios para su buen funcionamiento. En América Latina y el Caribe, la mayor parte de los países no han llegado a la etapa de integrar registros de cáncer en programas que funcionen plenamente, ya que todavía no tienen esos programas. Algunos registros suministran datos a instituciones académicas para fines de investigación, pero este propósito no justifica instituir registros nacionales. En estas circunstancias, el aprovechamiento más activo de datos de mortalidad, del uso de hospitales y de factores de riesgo puede ser más costo-efectivo que los registros para establecer prioridades y hacer planes estratégicos. Por el momento es más importante fortalecer los registros existentes que crear nuevos, pero los registros nuevos deben centrarse en apoyar el desarrollo de programas como los de detección de cáncer de cuello de útero y no en la investigación.

Diabetes. En la Región hay 30 millones de personas que sufren de diabetes, 13 millones de ellas en América Latina y el Caribe. Se predice que para 2010 esta segunda cifra habrá aumentado a 20 millones. Esta tendencia se acompaña de rápidos cambios culturales de dieta y estilo de vida complicados por el envejecimiento de las poblaciones. La diabetes es la causa principal de ceguera, amputaciones no traumáticas e insuficiencia renal. El costo de estas complicaciones es enorme, no solo en calidad de vida de los pacientes, sino también en gastos de atención de salud. Es especialmente preocupante que el número de casos de insuficiencia renal crece día tras día, pues pocos países de la zona pueden costear un acceso equitativo a diálisis y trasplantes.

Actividades del HCN en diabetes. Inicialmente se ha hecho hincapié en obtener consenso con la Asociación Latinoamericana de la Diabetes, la Asocia- ción Caribeña de la Diabetes y la Federación Internacional de la Diabetes (FID). En agosto de 1996 se llevó a cabo en Puerto Rico una reunión regional en la cual se redactó la Declaración de las Américas sobre Diabetes. Esta importante declaración constituye un plan estratégico regional, que preparó el camino para un proceso de planificación operacional y su ejecución. Ahora mismo se están organizando y consolidando los programas nacionales con el apoyo de la OPS, la FID e importantes donantes industriales.

Otras iniciativas de interés son la de programación integral de ENT basándose en la diabetes, que se está ensayando en el Caribe oriental; un curso regional de formación en epidemiología que se ofreció en 1996 en Colombia; apoyo a cursos regionales dictados por la FID para fortalecer asociaciones miembros; traducción al español de la publicación de la Federación Educación sobre diabetes: disminuyamos el costo de la ignorancia y redacción de un folleto titulado La diabetes en las Américas: datos para los profesionales de salud, el cual puede obtenerse si se solicita directamente al Programa HCN. Este programa ha conseguido fondos de la compañía Eli Lilly para demostrar en Chile las ventajas clínicas y económicas de educar a los pacientes de diabetes.

Causas externas: prevención de traumatismos. En América Latina y el Caribe los traumatismos están representados con $10 \%$ en la mortalidad general y con $18 \%$ en el número de años de vida potencial perdidos por discapacidad. Las causas externas originan $20,5 \%$ de los años de vida perdidos por hombres y $8,1 \%$, por mujeres. Las lesiones son la primera causa de muerte entre personas de 5 a 45 años de edad.

Al considerar los traumatismos o lesiones, se suelen dividir en intencionados y no intencionados. Estos últimos, que incluyen los accidentes de vehículos de motor, caídas, quemaduras y ahogamiento, ocurren dos veces más que los otros, pero los intencionados se están incrementando. Aun con recursos limitados, muchos países han logrado reducir los accidentes de vehículos con intervenciones efectivas y de bajo costo, por ejemplo designando calles de un solo sentido, poniendo nuevas señales de tráfico e impedimentos a la velocidad, pasando legislación sobre los cinturones de seguridad y lanzando promociones para la seguridad del tránsito. Todas las lesiones no intencionadas pueden disminuirse si la voluntad es firme. En cuanto a las intencionadas, los análisis realizados por el Programa indican que la violencia se rige por ciertos patrones que sugieren la posibilidad de hallar 
remedios estableciendo políticas apropiadas e intervenciones de salud pública.

Actividades del Programa HCN. Hasta la fecha el Programa se ha dedicado principalmente al análisis descriptivo de los datos regionales, información que se ha recogido en un documento, disponible en inglés y español, sobre la mortalidad debida a traumatismos en algunos países de las Américas. ${ }^{5}$ En cuanto a relaciones con otros organismos, el $\mathrm{HCN}$ se mantiene en contacto con el Programa de Promoción de la Seguridad y Prevención de Lesiones (SPI) de la OMS y con los centros colaboradores de la OMS en las Américas. Para la reunión del Consejo Directivo en 1996, el HCN y la Asociación Internacional de Lesiones Cerebrales (IBIA) hicieron una presentación conjunta acompañada de una exposición computadorizada sobre las lesiones encefálicas.

Otras enfermedades no transmisibles. Los trastornos neuropsiquiátricos producen más discapacidad que cualquier otra categoría de ENT: 30\% del total de años de vida potencial perdidos por discapacidad en América Latina y el Caribe. Entre las mujeres, el trastorno más común es la depresión y en los hombres, la dependencia del alcohol. Los problemas musculoesqueléticos, trastornos respiratorios crónicos y malformaciones congénitas producen 9,7 y $7 \%$, respectivamente, de los años de vida potencial perdidos por discapacidad, pero no se han abordado sistemáticamente en esas zonas.

$\mathrm{Al}$ intentar analizar la situación de las ENT en América Latina y el Caribe, se manifiesta en seguida la falta de datos. Para proceder con su cometido, el HCN necesita saber cuántos países han determinado su propia situación, cuántos han estimado la carga económica que representa, cuántos han desarrollado estrategias nacionales con metas, indicadores y medición de los resultados, qué recursos se han asignado a la prevención y el control de las ENT y si se están aplicando eficiente y efectivamente, cuán accesibles son los servicios, tratamientos y suministros para ENT a quienes los necesitan y si hay barreras que impiden ese acceso. En contraste con los países de Norteamérica, Europa, Asia Sudoriental y el Pacífico, en los de América Latina y el Caribe se han hecho muy pocos estudios de prevalencia bien diseñados sobre las ENT y sus factores de riesgo y complicaciones. Es

Roberts D. Mortalidad por lesiones no intencionales y violencia en las Américas: libro de referencia. Washington, DC: Organización Panamericana de la Salud; 1997. (Documento HCP/HCN 97.005, orig. inglés). necesario subsanar esta laguna para determinar qué intervenciones son necesarias y cómo iniciarlas.

Eficacia y efectividad de la prevención. Independientemente de la enfermedad de que se trate, todos los aspectos de prevención y control deben someterse a evaluaciones de eficacia y efectividad, y las ENT no son una excepción a la regla. La eficacia de la prevención se refiere al desempeño logrado con una ejecución total en condiciones ideales (figura 3). En cambio, se habla de efectividad de la prevención en cuanto a lo que se logra con las condiciones existentes de ejecución. Las siguientes prioridades del Programa de la OPS se justifican en parte por la eficacia de prevención posible. Así, las enfermedades cardiovasculares, que producen $45 \%$ de la mortalidad, pueden tener una eficacia de prevención superior a $50 \%$. El cáncer cervicouterino, principal sitio de tumores en la mujer, se puede prevenir en $90 \%$. En la diabetes mellitus, con 28 millones de casos en América Latina y el Caribe y un aumento proyectado de $40 \%$ para el año 2010, la eficacia de prevención sobrepasa 50\%. Los traumatismos causan $10 \%$ de la mortalidad, $18 \%$ de los años de vida potencial perdidos por discapacidad y su eficacia de prevención es de más de $40 \%$.

Los resultados que se obtienen en la prevención de enfermedad cardiovascular pueden ser tan espectaculares como los de Carelia del Norte, Finlandia, donde se logró una eficacia de prevención de

FIGURA 3. Eficacia estimada de las intervenciones preventivas para algunas enfermedades no transmisibles

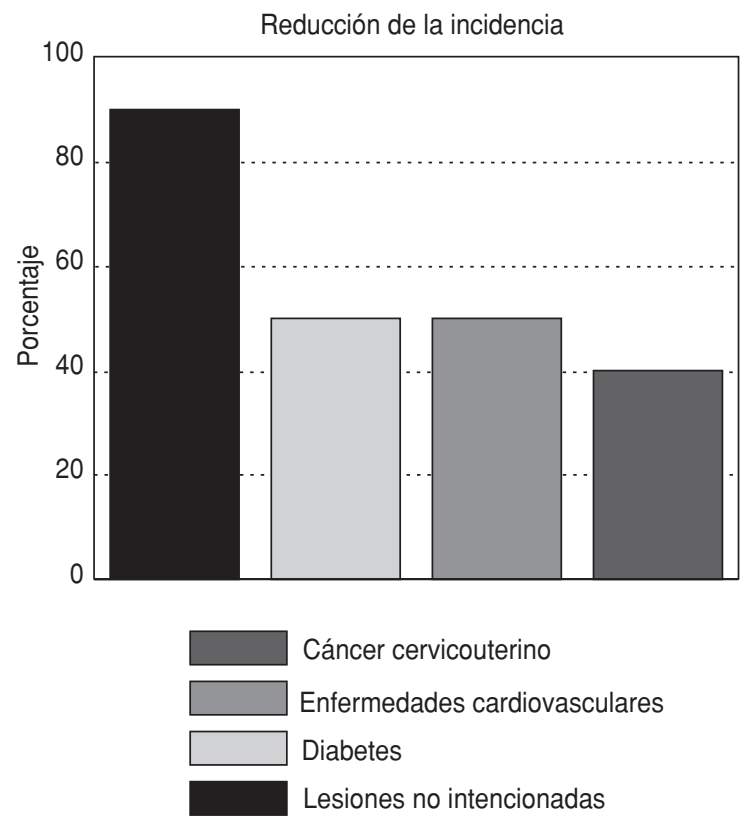


más de 50\%. En el período de 20 años de 1972 a 1992, la incidencia de cardiopatías en los hombres disminuyó $55 \%$ y en las mujeres, $68 \%$. Cabe subrayar que $80 \%$ de la reducción en los hombres y $72 \%$ de la reducción en las mujeres fueron atribuibles a la menor prevalencia de solo tres factores de riesgo: colesterol, hipertensión y hábito de fumar. En el Canadá, donde hace 5 años que se está llevando a cabo el Programa de Salud Cardiovascular de Nueva Escocia, ya se han registrado disminuciones en la prevalencia de los mismos tres factores de riesgo. Es muy probable, entonces, que en el Canadá se logren reducciones importantes de la incidencia y la mortalidad por cardiopatías al igual que en Finlandia.

No es solo en países industrializados donde tienen éxito las medidas de prevención, como prueban Mauricio y Cuba. En la primera isla se lanzó un programa contra las ENT a fines de los años ochenta y a los 5 años se habían registrado descensos de la prevalencia de algunos factores de riesgo, si bien era muy pronto para sacar conclusiones sobre los resultados. En Cuba, sin embargo, después de solo 4 años del proyecto de Cienfuegos la prevalencia de la hipertensión se ha reducido de 43,9 a $38,5 \%$.

Según los cálculos de la OMS, una media de disminución de $2 \mathrm{~mm}$ en la tensión arterial de una población resulta en una reducción de $6 \%$ en la mortalidad anual por accidentes cerebrovasculares, de $4 \%$ en la de enfermedad cardiovascular y de 3\% en la de todas las causas. Si se aplica este modelo a Cienfuegos, Cuba, donde la media de reducción de la tensión arterial ha sido $3,5 \mathrm{~mm}$, pueden esperarse descensos de la mortalidad respectiva de 9, 6 y 4,5\%. Actualmente, en los demás países de América Latina y el Caribe que no tienen programas integrados contra las ENT, la efectividad de la prevención de enfermedad cardiovascular oscilará con toda probabilidad entre 0 y $10 \%$. Los programas integrados dan los mejores resultados porque combinan actividades bien enfocadas de promoción de la salud con apoyo sistemático para la prevención clínica (que reduce la incidencia y las complicaciones), reorientación de los servicios para aumentar la calidad de la atención dirigida a la prevención secundaria (que reduce la tasa de complicaciones) y con una reevaluación constante del proceso, sus efectos y sus resultados.

En el Canadá y Finlandia ya hace unos 25 años que se emprendió la lucha contra el cáncer cervicouterino y con programas bien coordinados se han alcanzado eficacias de prevención de la incidencia de cáncer invasor de 70 y 90\%, respectivamente. El desempeño del Canadá es el mejor en toda la Región. En América Latina y el Caribe la efectividad de prevención varía de 0 a $15 \%$; Chile tiene el mejor porcentaje por el momento, con un programa bien diseñado que todavía no se ha ejecutado en toda su amplitud.

En Chile se ha puesto también en marcha un proyecto de prevención de la diabetes, para demostrar lo que puede conseguirse con una serie de actividades integradas. Entre estas se incluyen la educación de los pacientes y de los profesionales de la salud, atención mejorada de ciertos factores de pronóstico como el control metabólico y el cuidado de los pies, y participación de los pacientes en su propia atención de salud. Ese tipo de intervención ha dado resultados muy buenos en diversos países, en los que se ha podido apreciar la eficacia y la efectividad de programas de prevención de la diabetes. En Malmo, Suecia, y Da-Qing, China, la incidencia se redujo en $50 \%$ durante 5 a 6 años de seguimiento de personas en riesgo. En los Estados Unidos y el Canadá se han podido reducir en $60 \%$ las tasas de complicaciones debidas a la diabetes en poco más de 7 años que lleva en curso el Diabetes Controlled Clinical Trial.

En lo que se refiere a la prevención de traumatismos, la eficacia de la prevención deriva principalmente del estudio de las lesiones ocasionadas por accidentes de tránsito y oscila en general entre 40 y $50 \%$ en relación con la carga que representan en cada país. La efectividad de la prevención tal como se practica en América Latina y el Caribe puede estar entre 0 y $50 \%$, si bien algunos países como Trinidad y Tabago han experimentado reducciones de alrededor de $50 \%$ en el período de 10 años posterior a un plan estratégico para mejorar la seguridad del tráfico. Aun así, queda la posibilidad de mayores progresos y una eficacia potencial mucho más alta. En este campo, la aproximación epidemiológica (definir y medir el problema, identificar los factores de riesgo, intervenir y evaluar) requiere cooperación entre varios ministerios.

\section{Actividades del Programa HCN con los países}

Desde 1995, el Programa cuenta con un punto focal para comunicación y consulta en cada Estado Miembro de la OPS. También mantiene relaciones con un número de centros colaboradores de la OMS, especialmente en las áreas de diabetes, dolor en el cáncer, tamizaje, epidemiología de accidentes cerebrovasculares y establecimiento de políticas de estrategias integradas. Entre las organizaciones no gubernamentales con las que el Programa ha desarrollado relaciones oficiales, destaca como primera la FID y se han establecido también nexos de trabajo a nivel oficioso con la Asociación Internacional de Lesiones Cerebrales, la Fundación Interamericana del Corazón, La Liga Mundial de la Hipertensión y otras organizaciones. 
Desde sus comienzos, el Programa se ha ocupado de articular y diseminar políticas, planes y normas apropiadas para la Región. Producto de ello son documentos como la Declaración de las Américas sobre Diabetes y la adaptación de ideas que se han aplicado con éxito en otros lugares de mundo, como el programa Conjunto de Acciones para la Reducción Multifactorial de las Enfermedades No Transmisibles (CARMEN). La cooperación técnica se lleva a cabo por conducto de las Oficinas del Representante de la OPS en los países, con énfasis en el análisis de situaciones, determinación de prioridades, y respaldo de proyectos piloto. Para complementar esa labor, el HCN disemina información pertinente, ofrece capacitación a personal nacional y promueve la investigación. La movilización de recursos de cada país es un esfuerzo constante que fundamenta las actividades del Programa.

Vigilancia de las ENT. Para poder planear y evaluar intervenciones nacionales o regionales, es importante conocer las tendencias y los patrones de mortalidad y morbilidad correspondientes a las ENT. La vigilancia de los cambios exige crear sistemas de información o mejorar los sistemas de información existentes. El Programa utiliza tanto las estimaciones de mortalidad como las de discapacidad y está elaborando las de años de vida potencial perdidos para cada ENT.

Un aspecto interesante del trabajo del Programa es la vigilancia de factores de riesgo (hipertensión, hiperlipidemia, tabaquismo) y sus determinantes (grasa excesiva en la dieta, sedentarismo, actitudes) y su distribución. En América Latina y el Caribe hay poco conocimiento de esos factores y de la utilización de los servicios de prevención. Recientemente el Programa preparó normas para la recolección y el análisis estandarizados de datos sobre los factores de riesgo y los ha hecho circular para obtener comentarios al respecto. Se espera adaptar el documento para uso regional y posiblemente mundial.

Los proyectos CARMEN. El concepto fundamental de estos proyectos es combinar una promoción de salud estrictamente enfocada en factores de riesgo específicos con acciones médicas preventivas y servicios clínicos y un mejor tratamiento de los factores de riesgo secundarios.

Cada proyecto CARMEN requiere que el país asigne recursos para su ejecución. Esto por lo general es factible, ya que los proyectos representan principalmente una reorientación de los servicios de salud, lo que añade calidad y efectividad a los esfuerzos por reformar la atención de la salud. Los proyectos CARMEN son de alcance nacional pero tienen sitios de ejecución locales. La duración mínima es de 15 años, debido a que las intervenciones son relativamente complejas y los cambios en factores de riesgo y sus resultados tardan bastante tiempo en manifestarse. Las prioridades y la forma de abordarlas varían de un lugar a otro, pero todos los CARMEN precisan una evaluación de base y otras periódicas. El acuerdo entre CARMEN de la OPS y Country-wide Integrated Noncommunicable Disease Interventions (CINDI) [Intervención Territorial Integrada en Enfermedades No Transmisibles] de la OMS en Europa proporciona apoyo técnico mutuo.

En Valparaíso, Chile, se está llevando a cabo un proyecto CARMEN y en la Argentina se está diseñando otro. Los proyectos chileno y canadiense (Salud del Corazón de Nueva Escocia) son los primeros de una red. Actualmente, se está considerando para inclusión en la red CARMEN el proyecto de Cienfuegos, Cuba. También han expresado interés en participar en esa red de proyectos Costa Rica, México, Puerto Rico y Uruguay.

\section{Impacto de los programas en el campo de las enfermedades no transmisibles}

Si bien en todas las áreas de prevención de la salud media un intervalo entre las intervenciones y los resultados, esa transición dura más en el caso de las ENT que en el de las infecciones agudas y los traumatismos. De igual modo que las enfermedades no transmisibles toman tiempo para desarrollarse, también se necesita tiempo para reducir los factores de riesgo, ya que se originan en el comportamiento humano. Como ejemplo, baste decir que desde 1974 la prevalencia del hábito de fumar en el Canadá ha estado disminuyendo, pero solo $1 \%$ al año. La reducción de la carga de enfermedad es igualmente lenta y en Chile, que tiene un sólido programa nacional de lucha contra el cáncer cervicouterino, la disminución está ocurriendo también a $1 \%$ anual. De manera que así como los resultados se hacen esperar, la cooperación técnica aplicada al creciente peligro de las ENT también se ha desarrollado de forma relativamente lenta, en los últimos 30 años.

La respuesta regional y de los países todavía no ha llegado al punto que permita vaticinar un éxito rotundo, pero prácticamente todas las enfermedades incluidas en este grupo son susceptibles de prevención y control. Algunos países recién se están dando cuenta del impacto de estas enfermedades, mientras que otros ya están listos para actuar. La tendencia es positiva y el Programa de la 
OPS ya ha mostrado que tiene capacidad para dar apoyo estratégico, a pesar de que está gravemente limitado en recursos. Ciertamente es imperativo realzar la capacidad de la propia OPS para hacer frente plenamente a la misión del Programa $\mathrm{HCN}$, pues el desempeño en este campo será un viraje decisivo en el éxito de la reforma de los sistemas de salud. Las necesidades de salud y las prioridades de los países cambian rápidamente y la OPS tiene que anticipar y liderar el proceso a medida que se aproxima el nuevo siglo.

\section{SYNOPSIS}

Mindful of the increasing predominance of noncommunicable diseases in Latin America and the Caribbean, PAHO'S activities in this area are geared toward strengthening the ability of the Organization to support prevention and control initiatives in the Member States. This effort is led by the Program on Noncommunicable Diseases (HCN), which advises and assists the countries in the adoption of policies, strategies, and feasible and cost-effective programs for the prevention and control of chronic diseases of public health importance. Currently, the program is promoting integrated approaches to the prevention of major noncommunicable diseases (eg., the CARMEN project), that may include elements of the following priority areas: diseases of the circulatory system, diabetes, cancer of the uterine cervix, and injury prevention. In addition, pain management and palliative care are being carefully assessed as potential new areas for regional attention. This report presents updated figures for Latin America and the Caribbean, revealing the general morbidity burden attributable to these diseases, and also describes in detail the activities of HCN.

\section{BIBLIOGRAFÍA}

Canadian Task Force on the Periodic Health Examination. The Canadian guide to preventive health care. Ottawa, Canada: Canada Communication Group; c1994.

(The) Diabetes Control and Complications Trial Research Group. The effect of intensive treatment of diabetes on the development and progression of long-term complications in insulin-dependent diabetes mellitus. N Eng J Med 1993;329: 977-986.

Gribble JN, Preston SH, eds. The epidemiological transition: policy and planning implications for developing countries. Washington, DC: National Academy Press; 1993.

Hakama M. Screening for cervical cancer. En: Miller AB, ed. Advances in cancer screening. New York: Klumer; 1996.

Knowler WC, Narayan KM, Hanson RL, Nelson RG, Bennett PH, Tuomilehto J, et al. Preventing non-insulin-type diabetes. Diabetes 1995;44:483-488.

Llanos G, Libman I. Diabetes in the Americas. Bull Pan Am Health Organ 1994;28:285-301.

Murray CJL, Lopez AD, eds. Global comparative assessments in the health sector. Geneva: World Health Organization; 1994.
(The) Nova Scotia Health Survey 1995. Nova Scotia Department of Health; 1996.

Organización Panamericana de la Salud Educación sobre diabetes: disminuyamos el costo de la ignorancia. Washington, DC: OPS; 1996. (Comunicación para la salud 9).

Organización Panamericana de la Salud Condiciones de salud en las Américas. Edición de 1994, Vol 1. Washington, DC: OPS; 1994. (Publicación científica 549).

Organización Panamericana de la Salud. Número especial sobre cáncer de cuello de útero. Bol Oficina Sanit Panam 1996; 121:471-615.

Organización Panamericana de la Salud. Oncología clínica: manual de la American Cancer Society. 2a ed. Washington, DC: OPS; 1996. (Publicación científica 559).

Pan American Health Organization. Special issue on cancer of the uterine cervix. Bull Pan Am Health Organ 1996;30:283-420.

Puska P, Tuomilehto J, Nissinen A, Vartiainen E, eds. The North Karelia Project: 20 years, results, and experiences. Helsinki: National Public Health Institute and WHO Euro; 1995.
Robles SC, White F, Peruga A. Tendencias de la mortalidad por cáncer de cuello de útero en las Américas. Bol Oficina Sanit Panam 1996;121:478-490.

Robles SC, White F, Peruga A. Trends in cervical cancer mortality in the Americas. Bull Pan Am Health Organ 1996;30: 283-420.

Sharp I, ed. Coronary heart disease: are women special? London: National Forum for Coronary Heart Disease Prevention; 1994.

White F. La importancia del consenso en la prevención y el control de la diabetes en América Latina y el Caribe. Rev Asoc Latinoam Diabetes 1996;4:9-13.

White F. News from the WHO Regionsthe Americas. World Diabetes 1996;2(2-3).

White $\mathrm{F}$. The emerging international dominance of chronic diseases. Can I Public Health 1997;88:9.

White $\mathrm{F}$. The return of chronic disease. Foreign Affairs 1996;183(July-August).

World Health Organization. Hypertension control: report of a WHO Expert Committee. Geneva: WHO; 1996. (Technical report 862). 